\title{
Photoresponse of Inorganic-Organic Thin Film Composites Based on Chalcogenide Glasses
}

\author{
Andriy Kovalskiy ${ }^{\mathrm{a}}$, Maria White ${ }^{\mathrm{a}}$, Joshua Allen ${ }^{\mathrm{a}}$, Roman Golovchak ${ }^{\mathrm{a}}$, Justin \\ Oelgoetz $^{\mathrm{a}}$, Oleh Shpotyuk ${ }^{\mathrm{b}}$, Karel Palka ${ }^{\mathrm{c}, \mathrm{d}}$, Stanislav Slang ${ }^{\mathrm{d}}$ and Miroslav Vlcek ${ }^{\mathrm{d}}$ \\ ${ }^{a}$ Department of Physics and Astronomy, Austin Peay State University, 601 College Street, Clarksville, TN 37044, USA \\ ${ }^{b}$ Institute of Physics, Jan Dlugosz University, 13/15 Al. Armii Krajowej, Czestochowa, 42200, Poland \\ ${ }^{c}$ Department of General and Inorganic Chemistry, Faculty of Chemical Technology, University of Pardubice, Studentska \\ 573, 53210 Pardubice, Czech Republic \\ ${ }^{d}$ Center of Materials and Nanotechnologies, Faculty of Chemical Technology, University of Pardubice, nam. Cs. Legii \\ 565, 53002 Pardubice, Czech Republic
}

\begin{abstract}
Spin coated chalcogenide thin films have different structural and chemical properties than the films obtained by traditional methods such as thermal evaporation or sputtering. The solution-based method provides lower sensitivity of glass matrix to the influence of bandgap and superbandgap light. This property is very useful for non-linear optical applications based on high transparency of these materials in infrared spectral region. Arsenic selenide spin coated thin films were obtained by chemical dissolution of bulk glasses in ethylenediamine. The influence of preparation conditions, especially the annealing temperatures at the final stage of thin films synthesis, on in-situ kinetics of photodarkening (bleaching) at various energies and intensities of UV-VIS light was studied. It was found that at certain annealing conditions only transient photoinduced effects can be obtained by eliminating metastable kinetic component. Mechanisms of the photoinduced effects are discussed based on the parameters of relaxation functions.
\end{abstract}

Keywords: Chalcogenide Glass; Thin Films: Spin-Coating; Photoinduced Effects; Inorganic-Organic Composites

PACS: 78.66.Sq; 78.66.Jg; 81.07.Pr; 67.60.gj; 42.70.Ce; 42.70.Gi

\section{INTRODUCTION}

Thin films of non-oxide chalcogenide glasses (ChG - binary, ternary or multicomponent covalently bonded compounds based on chalcogen atoms $\mathrm{S}, \mathrm{Se}$, and Te and traditionally obtained by thermal evaporation method) are very promising materials for optics and photonics applications due to their high refractive index, great transparency in infrared spectral region and unique sensitivity of structure (and, consequently, optical properties) to bandgap or superbandgap light radiation [1-4]. However, such incompletely controlled high sensitivity to the UV and visible light is often very undesirable for infrared applications and can be suppressed either by post-technological treatment (temperature, light, or combination of both) or using other technological methods such as spin-coating of ChG dissolved in amine solvents with following thermal annealing in vacuum. The latter method has successfully been used to obtain thin films relatively photostable in UV-VIS region [5-8]. The structure of such films was revealed as a inorganic-organic composite where glass clusters are surrounded by organic residuals formed after thermal annealing [9-12].

In majority of previous studies of photo-induced effects in the $\mathrm{ChG}$ spin-coated thin films the conclusions about their poor photosensitivity were made based on the studies of arsenic sulfides irradiated with low or moderate light intensities of $10 \mathrm{~mW} / \mathrm{cm}^{2}$ or less. Our goal is, on the example of arsenic selenide binary compositions, to explore photoresponse of such thin films prepared at different technological conditions under higher intensities and different energies of exposing 
light and find correlation between the in-situ kinetics of photoinduced effects and possible mechanisms of photostructural transformations.

\section{EXPERIMENTAL PROCEDURE}

To obtain solution for spin-coating bulk glass was crushed and dissolved in ethylendiamine which serves as a good solvent for selenide glass. The usual time of dissolution at room temperature was 2-3 days in argon desiccant environment. The prepared solution is filtered and then static deposition technique at argon environment is performed onto silica substrate which was centrifuged at $1000+\mathrm{rpm}$. After that the samples are transferred to the furnace and annealed at different temperatures in nitrogen atmosphere. The best quality films were obtained by depositing from a $0.2 \mathrm{~g} / \mathrm{mL}$ solution. The thin films are then kept unexposed to ambient light in a desiccant.

Chemical composition of the spin-coated thin films were checked using high resolution X-ray Photoelectron Spectroscopy (XPS), Scienta ESCA-300, Lehigh University, USA and Energy Dispersive Spectroscopy data by Scanning Electron Microscope Hitachi 9300 FEGSEM.

Thin film composition $\mathrm{As}_{42} \mathrm{Se}_{58}$ was chosen to study the influence of technological conditions on in-situ kinetics of photoresponse in this type of materials. Using Swanepoel's method [13] thickness of the prepared films was found $\sim 1$ $\mu \mathrm{m}$. Samples were exposed with $385,530,780 \mathrm{~nm}$ LED light at high power $\sim 140 \mathrm{~mW} / \mathrm{cm}^{2}$. Transmission at the absorption edge region (corresponding to $\sim 20 \%$ transmittance) was tracked using Avasoft Dualspec spectrometer. The data were fitted to a stretched exponential function of the form:

$$
y=A_{1} \pm A_{2} e^{-\left(\frac{t}{T}\right)^{\beta}}
$$

Structure of the thin films before and after exposure was studied using Horiba Xplora Raman microscope with 785 nm laser as an excitation source.

\section{KINETICS OF PHOTORESPONE}

It is known that structure and properties of ChG thermally evaporated thin films are changing under exposure to bandgap and superbandgap radiation [1-4]. Such sensitivity is related to the difference between the structures of the thin films and bulk glasses, which are relatively photostable. The evaporation process creates significant excess of "wrong" and metastable homopolar bonds, which, together with high concentration of lone pair electrons of chalcogen atoms at the top of valence band, and additional free volume promote significant photostructural changes. Changes of optical properties of the thermally evaporated thin films are direct consequence of the mentioned structural changes under light exposure. At the same time, structure of spin-coated thin films of ChG is, according to Raman and XPS studies, much more similar to the structure of bulk glasses [12], and contains structural units of bulk glass linked by residual organic fragments. At the example of stoichiometric $\mathrm{As}_{2} \mathrm{~S}_{3}$ Fig. 1 clearly demonstrates high photosensitivity to light in the thermally evaporated thin film with a large metastable component and complete photostability of the spin coated film.
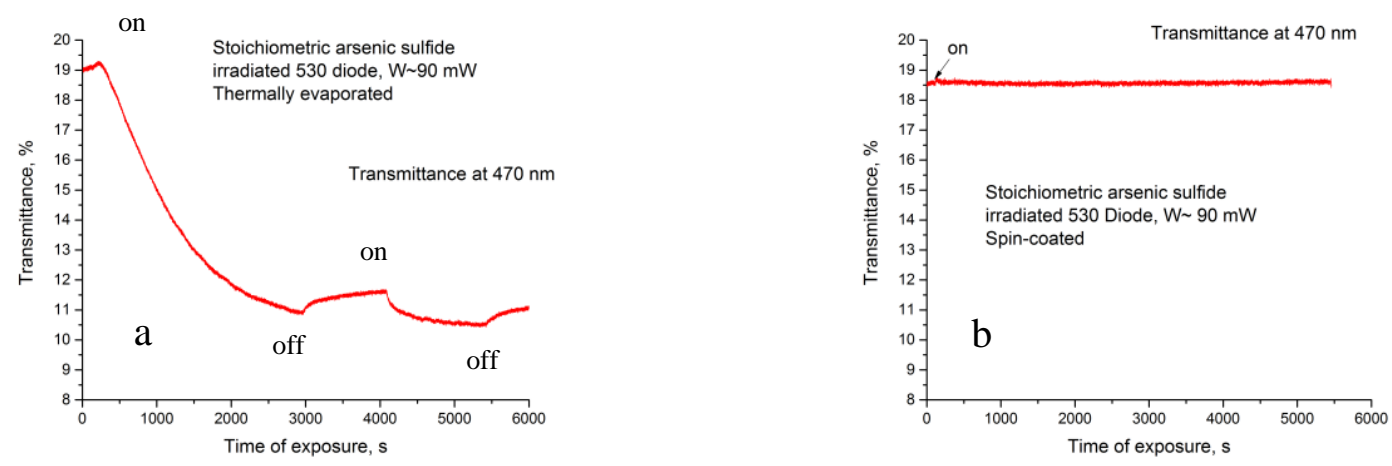

FIGURE 1. Kinetics of photoinduced change of optical transmittance at the absorption edge region in thermally evaporated (a) and spin coated (b) stoichiometric arsenic sulfide $\left(\mathrm{As}_{2} \mathrm{~S}_{3}\right)$ thin films at bandgap irradiation $\left(530 \mathrm{~nm}\right.$ diode, $\sim 90 \mathrm{~mW} / \mathrm{cm}^{2}$ power). 
Fig. 1a shows that after diode was on, the photodarkening (shift of optical absorption edge to higher wavelengths) of the thermally evaporated $\mathrm{As}_{2} \mathrm{~S}_{3}$ thin film takes place. The photodarkening shift can be partially reversed by annealing the thin film at the temperature just below glass transition. Thus irreversible and reversible parts of the effect (both can be named as a non-transient part) can be separated. There is also effect of change of transmittance in the on-off diode cycles which is called transient effect. The mechanisms of irreversible, reversible and transient effects are different and widely discussed in the literature [1-4]. .

Contrary to arsenic sulfide spin-coated thin films, the arsenic selenide spin-coated layers reveal weak transient photoresponse even at irradiation by subbandgap light (Fig.2). The magnitude of photoinduced shift of optical absorption edge strongly depends on energy of photons and light intensity, and increases with decrease of wavelength of the exposing LED.
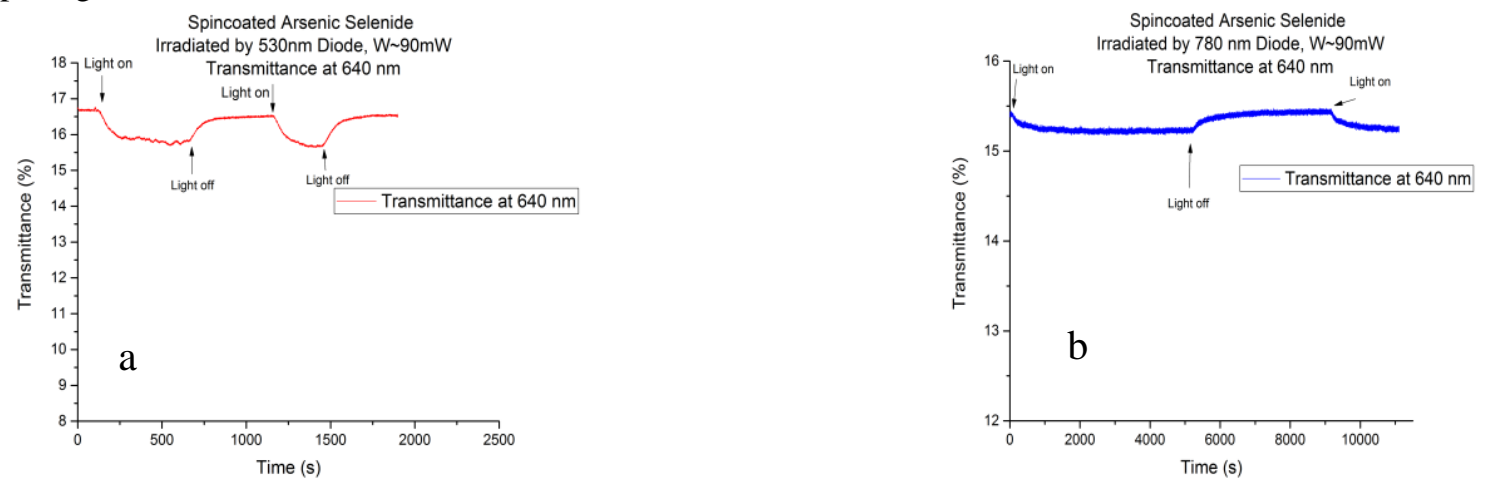

FIGURE 2. Kinetics of photoinduced change of optical transmittance at the absorption edge region in spin-coated arsenic selenide thin films exposed to LED with photon energy above (a) and below (b) optical bandgap.

In-situ kinetic behavior of optical transmittance at absorption edge in spin-coated $\mathrm{ChG}$ can be very different depending on technology of thin film preparation. Fig. 3 demonstrates that at certain annealing temperatures $\left(\sim 100{ }^{\circ} \mathrm{C}\right.$ for ethylendiamine solvent) the non-transient part can be completely eliminated and only stable with time transient part exist. Additionally, non-transient photodarkening in the films annealed at low temperatures is replace by photobleaching when annealed at temperatures close to the glass transition. Previous studies suggest structure of $\mathrm{ChG}$ spin-coated films can, in general, be considered as a fragments of bulk glass linked through the residual units of organic solvents [9]. The amount of the residual organic can be decreased with post-coating annealing changing the structure of the films. From other side, it is known [14] that irradiation promotes agglomeration in spin coated films and formation of several $\mathrm{nm}$ large encapsulated regions. Thus, at this point it can be concluded that the observed effects are related to the change in glass agglomeration and connection between them through organic residuals.

Fitting parameters of the in situ kinetics for the described transient part are given in Table 1. It is seen that the value

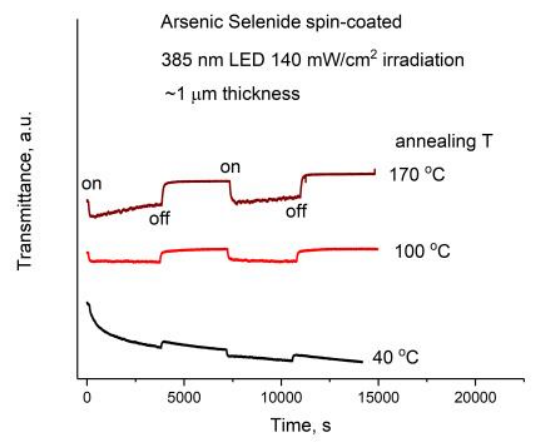

TABLE 1. Kinetic parameters for transient part of photodarkening and relaxation in spin-coated arsenic selenide thin films

\begin{tabular}{|c|c|c|c|c|c|c|c|c|}
\hline \multirow{3}{*}{ Sample } & \multicolumn{4}{|c|}{ Photodarkening } & \multicolumn{4}{|c|}{ Relaxation } \\
\hline & \multicolumn{2}{|c|}{$1^{\text {st }}$ Cycle } & \multicolumn{2}{|c|}{$2^{\text {nd }}$ Cycle } & \multicolumn{2}{|c|}{$1^{\text {st }}$ Cycle } & \multicolumn{2}{|c|}{$2^{\text {nd }}$ Cycle } \\
\hline & $\beta$ & $\tau, \mathrm{s}$ & $\beta$ & $\tau, \mathrm{s}$ & $\beta$ & $\tau, \mathrm{s}$ & $\beta$ & $\tau, \mathrm{s}$ \\
\hline Annealed $40^{\circ} \mathrm{C}$ & 0.74 & 90 & 0.80 & 30 & 0.92 & 31 & 0.84 & 27 \\
\hline Annealed $100^{\circ} \mathrm{C}$ & 0.90 & 36 & 0.95 & 40 & 0.65 & 44 & 0.69 & 45 \\
\hline Annealed $120^{\circ} \mathrm{C}$ & $\sim 1$ & 60 & $\sim 1$ & 73 & 0.99 & 74 & 0.90 & 74 \\
\hline Annealed $170^{\circ} \mathrm{C}$ & $\sim 1$ & 38 & $\sim 1$ & 60 & $\sim 1$ & 68 & 0.97 & 67 \\
\hline
\end{tabular}

FIGURE 3. Kinetics of photoinduced change of optical transmittance in spin-coated arsenic selenide thin films annealed at different temperatures 
of parameter $\beta$ increases with annealing temperature and the functions describing photodarkening and relaxation components of the transient optical effect are close to exponential. It should be mentioned that for the annealed samples we repeatedly achieved $\beta$ values slightly higher than 1 . We cannot completely assign such unusual data to the fitting error and further exploration is needed to understand possible relation of these values to the structural features of the spin-coated thin films.

\section{CONCLUSIONS}

It was found that optical transmission of arsenic selenide spin-coated thin films at the region of absorption edge is noticeably changing with time under the exposure to superbandgap high intensity LED light revealing both non-transient and transient effects. The non-transient part of the kinetics (photodarkening or photobleaching) strongly depends on the annealing temperature of the spin-coated thin films. At certain technological conditions it is possible completely eliminate non-transient part and observe only fully reversible transient effect (photodarkening) in multiple "on-off" cycles of light exposure. Fitting the photodarkening and relaxation curves using stretch exponential function shows tendency to pure exponential dependence at higher annealing temperatures.

\section{ACKNOWLEDGMENTS}

The team from Austin Peay State University acknowledges financial support from NSF RUI grant DMR-1409160 and NASA Tennessee Space Grant Consortium. Authors are thankful to Dr. Himanshu Jain from Lehigh University for support in XPS measurements. The group from Czech Republic appreciate financial support from project No. 16-13876S financed by the Grant Agency of the Czech Republic, from the grants LM2015082 and ED4.1.00/11.0251 financed by the MEYS of the Czech Republic and from ERDF project No. CZ.02.1.01/0.0/0.0/16_013/0001829

\section{REFERENCES}

1. A. Zakery and S. R. Elliott, J. Non-Cryst. Solids 330, 1-12 (2003).

2. Ke. Tanaka, Curr. Opin. Solid St. M. 1, 567-571 (1996).

3. A. Seddon, J. Non-Cryst. Solids 184, 44-50 (1995).

4. Ke. Tanaka and K.Shimakawa, Amorphous Chalcogenide Semiconductors and Related Materials, New York, Dordrecht, Heidelberg, London: Springer, 2011.

5. Y. Zou, H. Lin, O. Ogbuu, L. Li, S. Danto, S. Novak, J. Novak, J. D. Musgraves, K. Richardson and J. Hu, Opt. Mater. 12, 17231732 (2012).

6. S. Song, J. Dua and C. B. Arnold, Opt. Express 6, 5472-5480 (2010).

7. S. Shtutina, M. Klebanov, V. Lyubin, S. Rosenwaks and V. Volterra, Thin Solid Films 261, 263-265 (1995).

8. G. C. Chern, I. Lauks and K. H. Norian, Thin Solid Films 123, 289-296 (1985).

9. G. C. Chern and I. Lauks, J. Appl. Phys. 54, 2701-2705 (1983).

10. J .J. Santiago, M. Sano, M. Hamman and N. Chen, Thin Solid Films 147, 275-284 (1987).

11. J. Cook, S. Slang, R. Golovchak, H. Jain, M. Vlcek and A. Kovalskiy, Thin Solid Films 589, 642-648 (2015).

12. S. Slang, K. Palka, L. Loghina, A. Kovalskiy, H. Jain and M. Vlcek, J. Non-Cryst. Solids 426, 125-131 (2015).

13. R. Swanepoel, J. Phys. E Sci. Instrum. 16, 1214-1222 (1983).

14. T. Kohoutek, T. Wagner, M. Frumar, A. Chrissanthopoulos, O. Kostadinova, and S. N. Yannopoulos, J. Appl. Phys. 103, 063511 (2008). 